\title{
PIECEWISE LINEAR GROUPS AND TRANSFORMATION GROUPS
}

\author{
BY HERMAN GLUCK ${ }^{1}$
}

Communicated by George Mackey, September 23, 1968

The object of this note is to state certain theorems which show that the notions of topological group and transformation group become unreasonably restricted when transposed to the piecewise linear category. Details will appear elsewhere.

Let us understand a piecewise linear $(P L)$ group to be a topological group $G$ together with a piecewise linear structure on $G$ (i.e., triangulation of $G$ as a locally finite simplicial complex), in terms of which the group multiplication $G \times G \rightarrow G$ and inversion $G \rightarrow G$ are given by piecewise linear functions.

Theorem A. The only connected $P L$ groups are the abelian Lie groups, $\left(S^{1}\right)^{m} \times R^{n}$, and in general the only $P L$ groups are extensions of these by discrete groups.

This is an immediate consequence of

Lemma. Every $P L$ group is locally $P L$ isomorphic to the Euclidean group of the sume dimension.

The lemma is proved by choosing local $P L$ coordinates in $R^{n}$ for $G$, subdividing so that the multiplication map is linear on simplexes, and then examining the map in detail on an individual simplex.

TheOREM B. Two PL groups are PL isomorphic if and only if they are topologically isomorphic, and any topological isomorphism between them is automatically a $P L$ isomorphism.

This also follows easily from the above lemma.

Now let $G$ be a $P L$ group, acting as a topological transformation group on the $P L$ manifold $M$, via the map

$$
F: G \times M \rightarrow M \text {. }
$$

If $F$ is a $P L$ map, we say that $G$ is a $P L$ transformation group acting on $M$.

1 The author was partially supported during the preparation of this paper by a grant, GP 6895, from the National Science Foundation. 
TheOREM C. Let $G$ be a $P L$ transformation group acting on the $P L$ manifold $M$. If

(1) the action is effective,

(2) $\operatorname{dim} G \geqq 1$,

(3) $M$ is connected,

then $G$ has no fixed points (in the sense that no point of $M$ is left fixed by every element of $G$ ).

The proof proceeds by choosing local coordinates for both $G$ and $M$, subdividing so that the action map $F$ is linear on simplexes, and then examining $F$ on a top-dimensional simplex with a maximaldimensional face in the $G$ direction.

Theorem $\mathrm{C}$ says, among other things, that equivariant suspension of effective $P L$ actions of nondiscrete groups on spheres is not possible.

CoRollary 1. Let $G$ be an effective $P L$ transformation group acting on the connected $P L$ manifold $M$. Then the isotropy subgroup of each point of $M$ is discrete, and all orbits have the same dimension.

Corollary 2. Let $G$ be an effective $P L$ transformation group acting on the connected $P L$ manifold $M$. Then $\operatorname{dim} G \leqq \operatorname{dim} M$.

If $n \leqq m$, there is a standard action of $R^{n}$ on $R^{m}$ obtained by regarding $R^{n}$ as a subgroup of $R^{m}$ and letting it act on $R^{m}$ by translation.

THEOREM D. Let $G$ be an effective $P L$ transformation group acting on the connected $P L$ manifold $M$. Then there are local coordinates about the identuty in $G$, in terms of which the group operation is addition, and local coordinates about any preassigned point in $M$, in terms of which the action of $G$ on $M$ is standard.

This is proved by first choosing local coordinates about the identity in $G$ and about the preassigned point in $M$. Then a "flat area" is found on the orbit through that point and, using the piecewise linearity of the action, a local cross section is constructed through the flat area and slid back to the original point of $M$. Theorem $\mathrm{D}$ then follows directly.

\section{REFERENCES}

1. D. Montgomery and L. Zippin, Small subgroups of finite-dimensional groups, Ann. of Math. (2) 56 (1952), 213-247.

2. A. Gleason, Groups without small subgroups, Ann. of Math. (2) 56 (1952), 193212.

3. D. Montgomery and L. Zippin, Topological transformation groups, Interscience, New York, 1955.

University of Pennsylvania, Philadelphia, Pennsyluania 19104 